\title{
NUMERICAL SIMULATION OF AIRFOILS APPLIED TO UAVs
}

\author{
P. C. P. Corrêa \\ and M. N. D. Barcelos \\ ${ }^{a}$ Universidade de Brasília \\ Departamento de Engenharia Mecânica \\ Campus Universitário Darcy Ribeiro \\ S/N - Asa Norte, CEP. 70910-900 \\ Brasília, Distrito Federal, Brasi \\ paulocpcorrea@gmail.com \\ bUniversidade de Brasília \\ Departamento de Engenharia Mecânica \\ Campus Universitário Gama \\ Área Especial de Indústria Projeção A \\ Gama Setor Leste, CEP. 72444-240 \\ Brasília, Distrito Federal, Brasil \\ manuelbarcelos@gmail.com \\ Received: October 25, 2013 \\ Revised: November 21, 2013 \\ Accepted: December 21, 2013
}

\section{ABSTRACT}

This essay aims the process optimization when referred to aeronautical projects. By using mesh generators softwares and simulations made in CFD, the article employs numerical techniques to simulate airfoils and shows that is possible to extract accurated and conservative outcomes when compared to wind tunnel results. The test cases studied were based on the Selig 1223 type of airfoil and developed into the ANSYS platform, whereas by using the ICEM mesh tool, structured meshs were generated and imported to the CFX enviroment, where they could be simulated and analyzed.

Keywords: Airfoils, CFX, ICEM, Selig 1223

\section{NOMENCLATURE}

$\mathrm{C}_{\mathrm{L}}$ lift coefficient

\section{Greek symbols \\ $\alpha$ angle of attack}

\section{Subscripts \\ máx maximum}

\section{INTRODUCTION}

The UAV, unmanned aerial vehicle, is an aircraft that creates the needed lift to the flight by means of aerodynamic forces. The presence of pilots is not required, and it can also be radio-controlled with the need or not of human supervision, if its operation is fully automatic, the application of Programmable Logical Controlers (PLC) is necessary.

The concept of light, cheap, small, unmanned aircraft was first idealized for military purposes and has been gaining groung since I World War. In Brazil, its uses were evident in the last decade, when the civil purpose of recognizing and monitoring great areas became important for security reasons (Maj. Christopher, 1997).

Nowadays, in Brazil, the UAV has several different applications, which benefits by the association between enterprises and universities that provide fully national developed technologies.

The project missions are nothing more than objectives to be achieved that define the aircraft, which can be military or civilian. The work applications range from areas such as attack and rescue to monitoring and transportation.

Given the importance of UAV's for technological development, civilian or military, this article aims to facilitate the process of aircraft design.

The design of the entire aircraft is complex, and it's subdivided into smaller sets such as Aerodynamics, Structure, Stability and Propulsion. Depending on the kind of operation of the vehicle, it is understandeble that the aerodynamic design area has a degree of considerable importance, with the main task of selecting airfoils to be used on the wing and control surfaces.

The choice of shape to be used is based on its aerodynamic characteristics, namely: the amount of lift, the drag produced, and the momentum generated by it. The most conventional way to analyze these characteristics is through wind tunnel tests, a process extremely reliable, but with a high cost associated when dealing with small projects. Besides being an 
experimental procedure which requires data to be collected out over many trials, it is also necessary skilled labor and high cost equipment.

It is worth emphasizing that the wing design aimed, here, in this work has a wingspan of 3 meters and a root cord of 0.3 meters or so. Therefore, a wind tunnel capable of supporting a full size aerodynamic surface is difficult to build and too expensive to justify to fulfill the project requirements.

The implementation of a validated computational analysis can result in a faster and cheaper aerodynamic design without sacrificing reliability. The CFD, English short for Computational Fluid Dynamics, is nothing more than a numerical tool to analyze fluid-dynamic systems. This tool is based on the computational solution of the equations of mass, momentum and energy conservation that govern the flow.

The methodology to implement a CFD approach in the design framework consists of several steps. Primarily, it is performed the study and the development of the mesh to computationally represent the fluid using the platform ICEM-CFD. The construction of the mesh over the aerodynamic surface guarantying the quality of mesh elements ensures a realistic flow representation.

Next, it is defined the boundary conditions of the problem. They are basically related to the flow characteristics, if incompressible or compressible, if inviscid or viscous and if laminar or turbulent. Finally, the test cases are solved and the computer generated outcomes are post-processed by extracting the properties of interest on the aerodynamic surfaces. The test cases were based on the Selig 1223 airfoil geometry, because it is a shape with a wide experimental study available, what facilitates the comparison of results. The Reynolds number used in the simulations is about 300,000. In addition, comparisons of different turbulence models are executed in order to obtain the one that best approximates the experimental results, thus, bringing greater reliability to the process of computational analysis.

\section{NUMERICAL MODEL}

The equations solved in this work are the conservation of mass, momentum and energy, also known as Navier-Stokes equations. The NavierStokes equations are complemented by applying a turbulence model. The whole the of equations are discretized by using a finite volume method and by a RANS methodology through several time iterations in the CFX environment.

The definition of the proper problem modeling required the analysis different turbulence models, thus expecting to evidence their individual behaviors. The models that have been studied so far are: the SST and the K-epsilon.

The first turbulence model employed was the
Shear Stress Transport (SST), its choice was based on its accuracy for external flows over aerodynamic surfaces, characterized by being a hybrid model that combines the equations of k- epsilon and k-omega. In practical terms, the model works as the k-omega nearby the walls and as the k-epsilon in the most distant areas (Lars Davidson, 2006).

After, the k-epsilon turbulence model was defined by its accuracy in regions beyond the boundary layer, resulting in an excellent approximation, if the data to be extracted are related to Lift force component.

\section{METHODOLOGY}

The methodology for creating the mesh, first began by determining the geometry of the domain and the way the mesh can be constructed inside. The mesh creation process consisted basically of a multiblock method, in which the domain is split into nine distinct parts which are divided into three interconnected layers, also called a central block, four internal and four external.

The central region is adapted to the geometry of the airfoil so that when it is deleted, an empty region is devised and no mesh exists there, as can be seen in Figure 1. The following internal four blocks are dimensioned to adapt to the boundary layer, and the mesh is constructed with a high level of refinement in order to model as accurate as possible the behavior near the airfoil. Finally, the external blocks are defined with a lower level of refinement.

The strategy of separating the external blocks from the internal ones close to the airfoil is chosen because this arrangement makes possible to reduce the computational cost. The separation in blocks allows the choice of different element densities for the boundary layer and the outer region. This strategy would not work is a one block mesh is created, even assuming an exponential mesh growth from the airfoil to the freestream.

At the end of the mesh generation process, the whole domain has a continuous and consistent structured mesh.

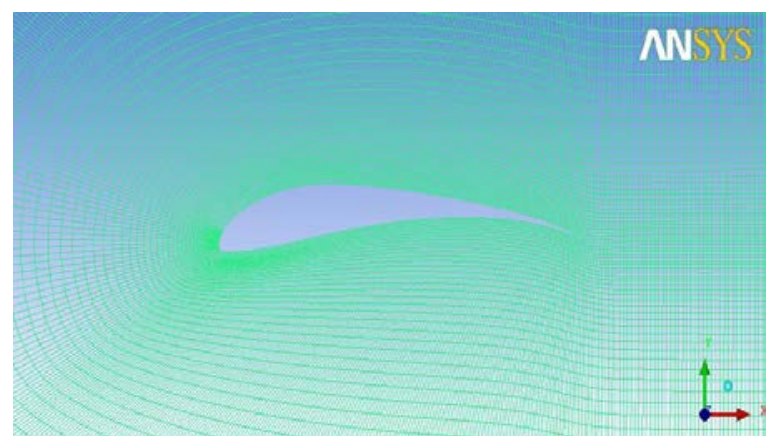

Figure 1. Mesh over the airfoil.

The whole process of creating the mesh was developed with the help of the pre-processing ICEM 
software, and as it belongs to the ANSYS platform, the generated mesh can be saved in files with a portable format and can be used in any numerical simulation software, not only the ones from the ANSYS packge. Here, the CFX software is preferred because it is well known in the scientific and technical communities and also belongs to the ANSYS platform.

\section{BOUNDARY CONDITIONS}

In Fig. 2, it showed how the conditions were applied to the domain, and they are divided as follows: the input (Inlet) conditions defined throughout the portion of arrows directed to model, the output conditions (Outlet) defined by the portion of arrows leaving the model, the conditions at the wall defined on the body shape, and finally the symmetry conditions defined on the faces perpendicular to the z-axis.

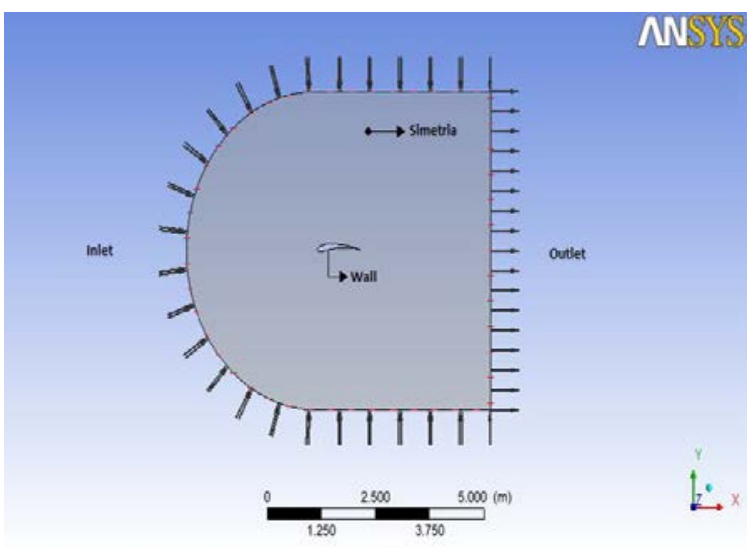

Figure 2. Representation of airfoil's boundary conditions.

For the numerical analysis, it was assumed a turbulent, isothermal and incompressible flow, and the physical parameters were defined for air at $25^{\circ} \mathrm{C}$. The simulation was also planned to truncate after the thousandth iteration or after reaching a residual value of at least $10-8$.

In the turbulence models, the magnitude of the turbulence parameters were defined as an average of 0.037 , as suggested by the manual of the software when simulating external flows of this sort.

\section{RESULTS AND DISCUSSION}

Defined all initial parameters, the CFX-Solver solves iteratively the equation model. This process was repeated for every different angle of attack, using SST and k-epsilon turbulence models.

It is expected that the results resemble as much as possible the values recovered over experimental works. For a better view of this behavior, it is usual to plot the curve " $\mathrm{Cl} \times \alpha^{\prime}$ collected for different points, curves which can be observed and compared in Figure 3.

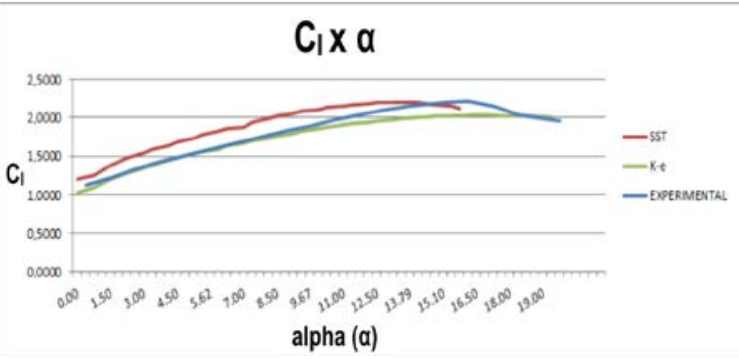

Figure 3. Lift versus angle of attack for experimental data and numerical simulations using the SST and képsilon turbulence models $(\mathrm{Re}=302200)$.

Ever more is remarkable the participation of computational methods in aerodynamics, an example is the market for unmanned aerial vehicles (UAVs). This increasing demand causes a need to develop efficient CFD methodologies to obtain more accurate results.

The judgment if a numerical result is valid or not depends always on the quality of reference data that is used to compare to, and also numerical methodology employed is reliable or not. The proposal of this work is develop a design methodology by using numerical tools with a compromise between high fidelity and time spent for modeling and solution of the problem. Therefore, in this work was chosen to use the full Navier-Stokes equations augmented by turbulence models for a quasi-2D domain, keeping a compromise. The parameter which are considered as reliable for the comparison in the article are the experimental data of the Selig 1223 airfoil provided by the site of UIUC Applied Aerodynamics Group of the University of Illinois.

After the treatment of the results one can notice a clear similarity between the numerical and the experimental data. The curve for the turbulence SST model approximates the value of the maximum lift coefficient, before the stall, a value of considerable importance in the development of aeronautical projects. However, the results obtained by the kepsilon model represent with certain accuracy the experimental data in the first half of the analysis and subsequently present a more conservative behavior.

So far, the goal of recovering the experimental behavior via CFD simulations was reached. At this moment, a specific study of mesh refinement and better adaption of the model to the quasi-2D domain are being executed to improve the fidelity of the simulation results. Part of this study has as goal to achieve a more detailed analysis of the boundary layer, by determining the best mesh density that better represents this region. The second part of the study has as goal to change some parameters of the turbulence models to adapt then to the use of quasi2D domains, enhances accuracy of prediction of the drag. 
Another study that will be done in parallel is to devise a method to determine the lift and drag coefficients of a wing from the values found for its cross-section, values that must be obtained by numerical computational simulations, based on CFD tools.

\section{ACKNOWLEDGEMENTS}

The authors would like to thank the University of Brasília for providing the resources necessary to the research.

\section{REFERENCES}

Davidson, L., 2006, An Introduction to Turbulence Models. Chalmers University of Technology, Göteborg, Sweden.

Christopher, M., and Jones, A., 1997, Unmanned Aerial Vehicles (UAVs) an Assessment of Historical Operations and Future Possibilities, The Research Department Air Command and Staff College. 